\title{
All-reflective ring illumination system for photoacoustic tomography
}

Suhail Salem Alshahrani

Yan Yan

Naser Alijabbari

Alexander Pattyn

Ivan Avrutsky

Eugene Malyarenko

Joemini Poudel

Mark Anastasio

Mohammad Mehrmohammadi 


\title{
All-reflective ring illumination system for photoacoustic tomography
}

\author{
Suhail Salem Alshahrani, ${ }^{a}$ Yan Yan, ${ }^{a}$ Naser Alijabbari, ${ }^{a}$ Alexander Pattyn, ${ }^{a}$ Ivan Avrutsky, ${ }^{b}$ Eugene Malyarenko, ${ }^{c}$ \\ Joemini Poudel, ${ }^{\mathrm{d}}$ Mark Anastasio, ${ }^{\mathrm{d}}$ and Mohammad Mehrmohammadi $\mathrm{i}^{\mathrm{a}, \mathrm{b}, \mathrm{c}, *}$ \\ aWayne State University, Department of Biomedical Engineering, Detroit, Michigan, United States \\ 'Wayne State University, Department of Electrical and Computer Engineering, Detroit, Michigan, United States \\ 'Barbara Ann Karmanos Cancer Institute, Detroit, Michigan, United States \\ 'Washington University in St. Louis, Biomedical Engineering Department, St. Louis, Washington, United States
}

\begin{abstract}
Given that breast cancer is the second leading cause of cancer-related deaths among women in the United States, it is necessary to continue improving the sensitivity and specificity of breast imaging systems that diagnose breast lesions. Photoacoustic (PA) imaging can provide functional information during in vivo studies and can augment the structural information provided by ultrasound (US) imaging. A full-ring, all-reflective, illumination system for photoacoustic tomography (PAT) coupled to a full-ring US receiver is developed and tested. The US/PA tomography system utilizes a cone mirror and conical reflectors to optimize light delivery for PAT imaging and has the potential to image objects that are placed within the ring US transducer. The conical reflector used in this system distributes the laser energy over a circular cross-sectional area, thereby reducing the overall fluence. This, in turn, allows the operator to increase the laser energy achieving better cross-sectional penetration depth. A proof-of-concept design utilizing a single cone mirror and a parabolic reflector is used for imaging cylindrical phantoms with light-absorbing objects. For the given phantoms, it has been shown that there was no restriction in imaging a given targeted cross-sectional area irrespective of vertical depth, demonstrating the potential of mirror-based, ring-illuminated PAT system. In addition, the all-reflective ring illumination method shows a uniform PA signal across the scanned cross-sectional area. ๑ The Authors. Published by SPIE under a Creative Commons Attribution 4.0 Unported License. Distribution or reproduction of this work in whole or in part requires full attribution of the original publication, including its DOI. [DOI: 10.1117/1.JBO.24.4.046004]
\end{abstract}

Keywords: full-ring illumination; omnidirectional; conical mirror; ultrasound tomography; photoacoustic tomography; ring ultrasound transducer.

Paper 190017R received Jan. 18, 2019; accepted for publication Apr. 8, 2019; published online Apr. 26, 2019.

\section{Introduction}

The combined ultrasound (US) and photoacoustic (PA) tomographic imaging system described in this paper has broad imaging applicability. With its full-ring US receiver and illumination source, one potential application could be breast imaging. Breast cancer is a common cancer type among women and is a major health concern affecting many lives worldwide. In 2018, it was estimated that the number of newly diagnosed breast cancer cases around the world will be above 2 million. ${ }^{1}$ Mammography, magnetic resonance imaging (MRI), and B-mode US are three of the most common imaging modalities used for breast cancer screening, ${ }^{2,3}$ and each has its unique shortcomings. Mammography has low sensitivity in detecting breast lesions in women with high-density breast tissue, which is critical since this population is considered to be at a higher risk of developing breast cancer. ${ }^{3,4}$ MRI can be used in conjunction with mammography to detect breast tumors in dense breasts. ${ }^{5,6}$ However, the availability and cost of MRI imaging restrict the accessibility of this modality. Conventional B-mode US is one of the most widely used medical imaging techniques for screening various types of human tissues, and it is a highsensitivity, non-ionizing, and low-cost tool that can produce images in real time. ${ }^{7,8}$ Yet its low specificity in breast screening can lead to unnecessary biopsies. ${ }^{9,10}$ Therefore, it is essential to

*Address all correspondence to Mohammad Mehrmohammadi, E-mail: mehr@ wayne.edu develop new or complementary breast cancer imaging modalities that minimize or eliminate the existing limitations.

Ultrasound tomography (UST), employing a ring-shaped US transducer, has shown promise for breast cancer screening. ${ }^{11-15}$ Moreover, PA imaging has demonstrated potential in detecting carcinomas $^{16,17}$ and angiogenesis due to tumor growth. ${ }^{18,19}$ The addition of photoacoustic tomography (PAT) to the UST imaging can enhance a physician's diagnostic capability by providing functional information about the tissue of interest. Moreover, PA imaging can be easily integrated with UST since the two modalities share the same acquisition hardware. Previous PA/ PAT visualization tools ${ }^{20-24}$ either suffer from the use of inefficient illumination methodologies or distort the breast tissue, reducing tissue circulation which may affect the PA results.

A significant challenge for PAT breast imaging is providing sufficient fluence for the desired cross section, while avoiding the maximum permissible exposure (MPE) limit for the tissue. For a full-ring illumination system, expanding the illumination area will help deliver a higher laser energy per pulse to the targeted cross-sectional slice, while keeping fluences below the American National Standards Institute limits. ${ }^{25}$ For example, a $10-\mathrm{mm}$ diameter laser beam, with $200-\mathrm{mJ} /$ pulse energy will result in fluence of $253 \mathrm{~mJ} / \mathrm{cm}^{2}$. However, using the coneshaped reflector and the parabolic reflector to create the omnidirectional ring illumination pattern, with a 5-mm thickness and a $10-\mathrm{cm}$ diameter cross-sectional area, will result in a fluence of $12.7 \mathrm{~mJ} / \mathrm{cm}^{2}$. In this example, the fluence of the full-ring illumination system is about 20 times lower than that of direct 
illumination, and it is below the MPE limit which is $20 \mathrm{~mJ} / \mathrm{cm}^{2}$ for $532 \mathrm{~nm}$.

The full-ring illumination system has been used in other applications by deploying either a cone mirror or conical lens combined with an acoustically penetrable optical reflector (APOR) ${ }^{26-28}$ However, the APOR's low power threshold and acoustical transparency make it an impractical solution for full-ring illuminated systems. At the same time, the transmission coefficient of the APOR is affected by the incidence angle of the acoustical signal, ${ }^{26}$ so a $45-$ deg APOR, which should be used in this prototype, will have about $65 \%$ US transmittance. Conversely, any design using lenses to create a ring beam ${ }^{29,30}$ reduces the wavelengths available for spectroscopic imaging due to possible chromatic aberrations. To overcome earlier limitations, the full-ring US/PA tomography system using omnidirectional optical reflectors is proposed, which can deliver the needed energy for imaging and is vertically translatable to image the entire breast. ${ }^{31-33}$

The all-reflective, ring illumination PAT system proposed in this paper provides a practical, easily scalable, low fluence imaging system, capable of imaging tissue-mimicking phantoms with significant depth. The three-mirror system presented in this paper is capable of dispersing the light energy over a greater area to reduce the energy fluence, and in the process illuminates more of the tissue which overcomes the light penetration limitations for PAT imaging. In the first two experiments, a ring illumination source was used with a linear US transducer to study the efficiency of full-ring illumination in tissue-mimicking phantoms. In the final experiment, a proof-of-concept PAT imaging prototype consisting of a cone-shaped reflector, a large parabolic reflector, a tunable laser source, and a UST engine equipped with a ring US transducer, was used to image a tissue-mimicking phantom. The data generated using this system are presented with an eye toward laying the groundwork for a future three-mirror system with near-normal tissue illumination and even greater imaging depth.

\section{Material and Methods}

\subsection{Development and Validation of Ring-Illumination Optical System with Linear Array Acquisition}

The performance of the ring illumination mode was evaluated with regard to the PA imaging depth using two tissue-mimicking phantoms made of $10 \%$ porcine gelatin (G2500, SIGMAALDRICH, Missouri) and $0.2 \%$ cellulose (S5504, SIGMAALDRICH, Missouri). For the first experiment, a $700-\mu \mathrm{m}$ diameter graphite absorber was inserted obliquely into a gelatin phantom, and the PA signal was measured with respect to the horizontal depth [Fig. 1(a)]. The illumination source for the experiment was a pulsed $532 \mathrm{~nm}$, Nd:YAG, 8-ns laser (Quanta-Ray Pro 270, Spectra-physics, California) with an output of $10 \mathrm{~mJ}$ per pulse. A $100-\mathrm{mm}$ diameter parabolic mirror (45-944, Edmund Optics, New Jersey) and cone-shaped reflector (68-791, Edmund Optics, New Jersey) were used to create the ring beam by adjusting the distance between the cone mirror and the parabolic ring reflector [Fig. 1(b)]. For the second experiment, a 70-mm diameter gelatin phantom containing two graphite absorbers with $500-\mu$ m diameters were placed in two planes [Fig. 1(c)] and imaged to determine the maximum imaging depth and the plane selectivity of the full-ring illumination method. Large scale parabolic reflector (P19-0300, Optiforms Inc., California) with 243-mm diameter was utilized with a cone-shaped reflector to create a large ring-shaped beam with 4-mm thickness. A 532-nm laser source (PhocusCore, Optotek, California) was used, resulting in $4.77 \mathrm{~mJ} / \mathrm{cm}^{2} \mathrm{flu}-$ ence in the targeted cross-sectional area. In this experiment, it was not possible to adjust the position of the ring beam, so the beam was $17 \mathrm{~mm}$ below the targeted cross-sectional area [Fig. 1(c)]. In both experiments, a programmable US scanner (Vantage 128, Verasonics Inc., Washington, USA) was utilized with an L11-4 linear array transducer, operating at $8.4 \mathrm{MHz}$ center frequency, for US and PA signal acquisition.

\subsection{Photoacoustic Tomography Using Full-Ring Illumination and Ring-Shaped Transducer}

The full-ring US transducer and illumination system, shown in Fig. 2(a), also used a gelatin phantom for system characterization. In this experiment, an $89 \mathrm{~mJ} /$ pulse laser source (PhocusCore, Optotek, California, USA) was expanded to a ring shape, resulting in $4.7 \mathrm{~mJ} / \mathrm{cm}^{2}$ of fluence at $532 \mathrm{~nm}$. The ring US transducer has a 200-mm inner diameter and comprised 256 elements, with an element pitch of $2.45 \mathrm{~mm}$ and a height of $9 \mathrm{~mm}$. In PAT mode, the scattered signals were recorded by all 256 elements at a sampling rate of $8.33 \mathrm{MHz}$. The 243-mm diameter parabolic reflector and the cone-shaped reflector (a)

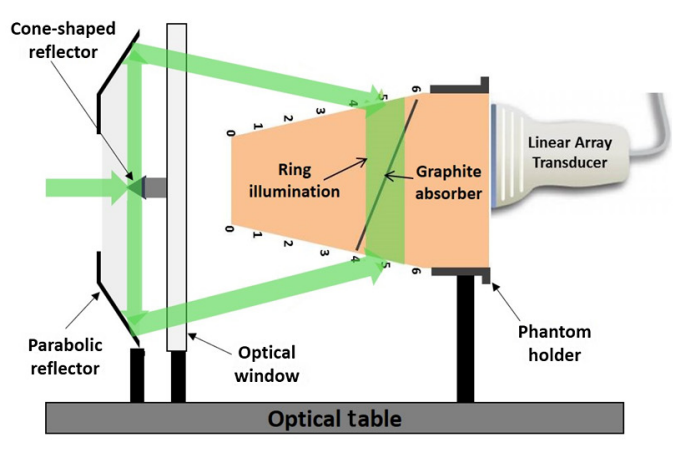

(b)

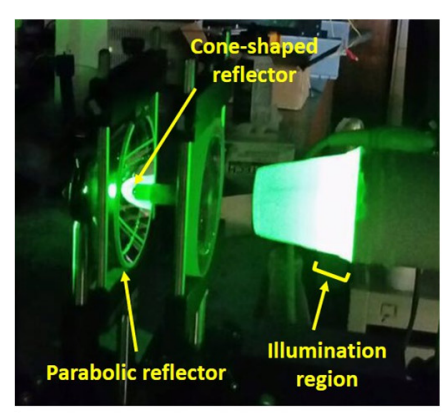

(c)

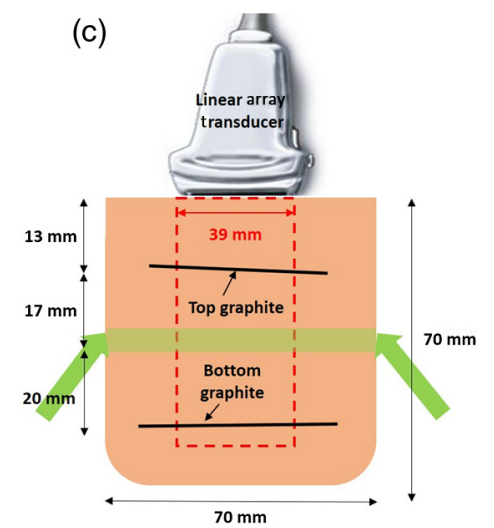

Fig. 1 (a) Experimental setup of the breast phantom embedded with a diagonal graphite absorber and (b) the photograph of the same experimental setup. (c) Diagram showing the dimensions of the tissuemimicking phantom used for the second experiment. The US scanning area is enclosed in the red dashed lines, and the ring beam has fallen $17 \mathrm{~mm}$ below the targeted cross section. 
(a)

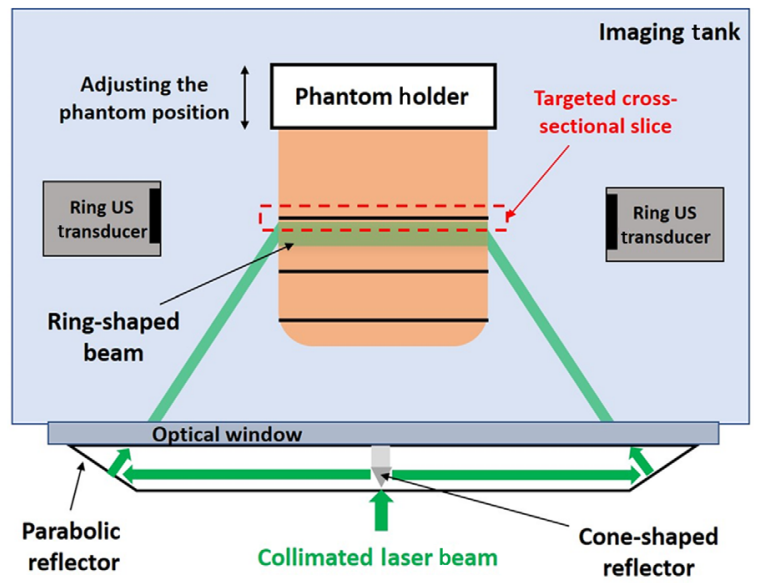

(b)

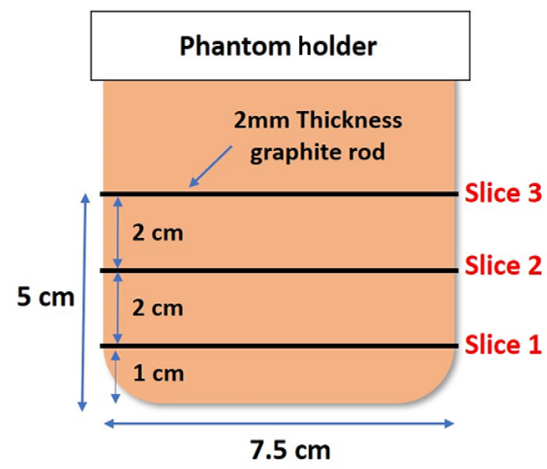

Fig. 2 (a) The setup for the full-ring illumination and full-ring acquisition experiments. The cone-shaped mirror and the parabolic reflector create the ring-shaped beam for the scanned cross section. The ring beam is positioned $10 \mathrm{~mm}$ below targeted cross-sectional slice for the discussed experiments. (b) The diagram shows the dimension of the tissue-mimicking phantom and the position of the graphite rods from the bottom slice (slice 1) to the top slice (slice 3).

were used to create the ring-shaped beam with 4-mm thickness on the phantom surface. The phantom used for this experiment was $7.5 \mathrm{~cm}$ in diameter and made of $12 \%$ porcine skin gelatin (G2500, SIGMA-ALDRICH, Missouri) mixed with $0.4 \%$ cellulose (S5504, SIGMA-ALDRICH, Missouri). Three graphite rods, with 2-mm thickness, were placed horizontally inside the phantom in three layers [Fig. 2(b)]. The ring beam was adjusted on each cross-sectional slice by translating the phantom in the vertical direction. The results from this experiment demonstrated the uniqueness of the all-reflective PAT system in creating co-registered PA-US tomographic images with significant depth and will be discussed further in the results section.

\section{Results and Discussion}

\subsection{Photoacoustic Results Using Full-Ring Illumination with a Linear Array Transducer}

Figure 3 plots the PA image for the diagonal graphite absorber in gelatin for the experimental setup shown in Fig. 1(a). A clear PA signal is detected within the field of view of the linear array US

(a)

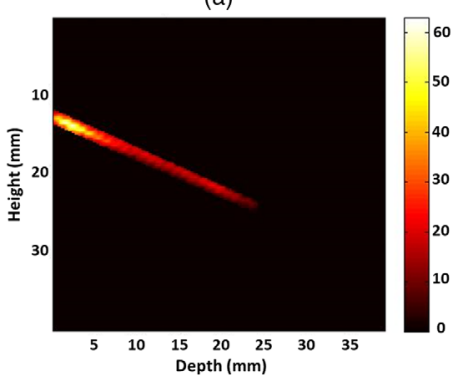

Fig. 3 (a) The PA signal distribution across the illuminated crosssectional slice of the gelatin phantom. (b) The graph demonstrates a normalized PA amplitude versus depth. A uniform PA signal amplitude is seen between the depths of 10 and $20 \mathrm{~mm}$. transducer, and a depth of $25 \mathrm{~mm}$ inside the phantom is imaged. As anticipated, the PA signal intensity is the largest superficially and is attenuated with horizontal depth [Fig. 3(a)] due to absorption and scattering. The normalized PA amplitude versus horizontal depth for this image is plotted in Fig. 3(b), demonstrating the depth dependence of the fluence and the relative uniformity of the PA signal within the middle portion of the phantom. The uniform PA signal between 10 and $20 \mathrm{~mm}$ underscores the effectiveness of omnidirectional ring illumination in providing a uniform fluence map within a targeted cross section. In an ideal PA platform, the fluence is depth-independent, and the proposed ring illumination system (Sec. 3.3) can enhance the PAT imaging by providing a more uniform illumination map. The PA signal after $25 \mathrm{~mm}$ disappears as the angled optical absorber moves outside of the selected illuminated cross-sectional area.

Figure 4 plots the results from the second experiment, which used two graphite absorbers in separate planes, as shown in Fig. 1(c). Since the linear array transducer has a limited field of view, only a section of the horizontal graphite absorber is shown in Fig. 4(a). As anticipated, both objects can be seen in the US image, while in the PA image, the targeted layer shows a high PA signal which demonstrates the selectivity of the full-ring illumination mode in imaging a targeted crosssectional slice. The full-ring illumination is also able to deliver sufficient energy to the core of the 70-mm tissue-mimicking phantom [Fig. 4(b)]. This finding highlights the benefit of the ring-illumination system which allows for a lower fluence and a greater cross-sectional illumination depth. The limited field of view of the linear transducer used in these experiments would also not be a problem for the full-ring US transducer, where all objects would be visualized regardless of the location.

It is important to mention that there was an observed PA signal from the middle layer of the phantom (at a depth of $30 \mathrm{~mm}$ ) which happened due to two reasons. First, the phantom was created in two parts by initially pouring the first layer and then allowing it to set so it could support the graphite rod, after which a second layer of the same material was poured to finish 
(a)

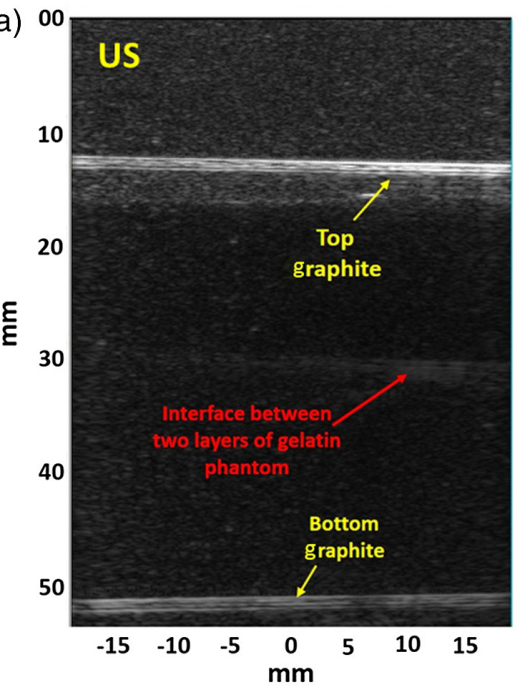

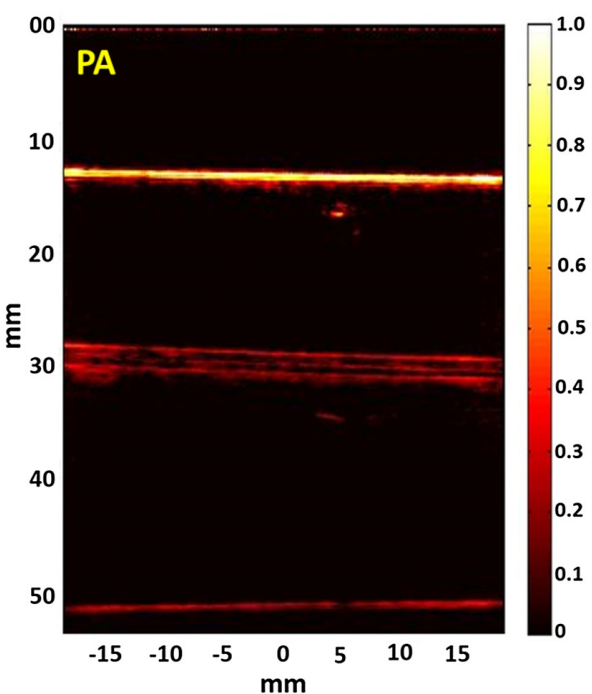

(b)

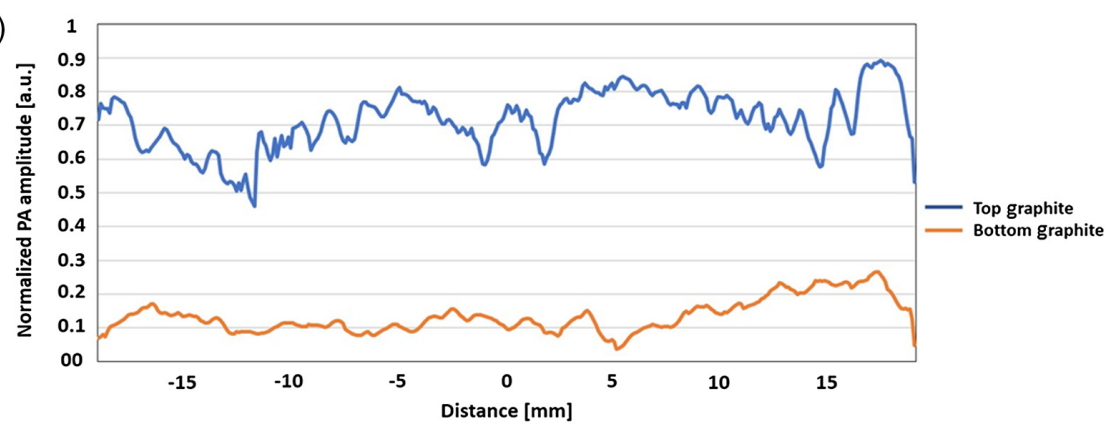

Fig. 4 (a) The US image (left) showing the graphite absorbers in two different planes. The interface seen in the picture is an artifact of the phantom-making process. The PA image (right) shows the top and the bottom graphite absorbers, with a more visible top object. (b) The plot of the normalized PA signal amplitude across the top and bottom graphite absorbers. The targeted, top graphite absorber has a larger PA signal amplitude.

the phantom. The setting process of the first layer and the possible settling of the cellulose scattering material could have created the PA signal seen at the interface. The second reason is related to the incident position of the ring beam on the interface surface between two layers of gelatin phantom which is located at $20 \mathrm{~mm}$ above the bottom graphite.

\subsection{Large-Scale Omnidirectional Illumination for Full-Ring Photoacoustic Tomography Experiment}

Figure 5 shows the UST and PAT images of the full-ring tomography system shown in Fig. 2(b). The UST images are reconstructed using the waveform method, ${ }^{34}$ and PAT images are reconstructed using the backprojection method. ${ }^{35}$ For the backprojection reconstruction, the RF signals received at each transducer across multiple acquisitions of the same slice are averaged together to increase the signal-to-noise ratio (SNR). In Fig. 5, the top and bottom cross-sectional slices, which are separated by $4 \mathrm{~cm}$ of gelatin, are shown. In general, the results demonstrate the vertical depth-independent imaging capability of the full-ring illumination system utilizing a ring US transducer.

The normalized PA amplitude as a function of the horizontal depth for the three different cross sections is shown in Fig. 6. As anticipated, all three slices showed high PA signal close to the outer surface, and the signal degraded slightly as the horizontal depth increased. In general, the results once again showed the deposited energy uniformity within the phantom, irrespective of vertical imaging depth.

To increase the fluence in the central part of the scanned slice, it is important to improve the incident angle of the ring beam and the incident location of the ring beam with respect to the targeted cross-sectional area. In the previous experiment, the ring beam was positioned $10 \mathrm{~mm}$ below the targeted cross-sectional slice. Future works will examine the effects of target illumination as a function of beam position below a cross-sectional area and the resulting PA image quality.

\subsection{Future Work: An Optimal, Adjustable, All-Reflective, Full-Ring Illumination Photoacoustic Tomography System}

For the full-ring illumination and US transducer system, the single cone mirror and parabolic reflector imaging system is able to create a ringed beam with a 39-deg angle with respect to the phantom surface. To improve the efficiency of the illumination, it is important to enhance the incident angle of the ring beam. The proposed three mirror system (Fig. 7) will have a tissue incidence angle of 66-deg with respect to the object surface, as opposed to 90 -deg or normal incidence, which is anticipated 

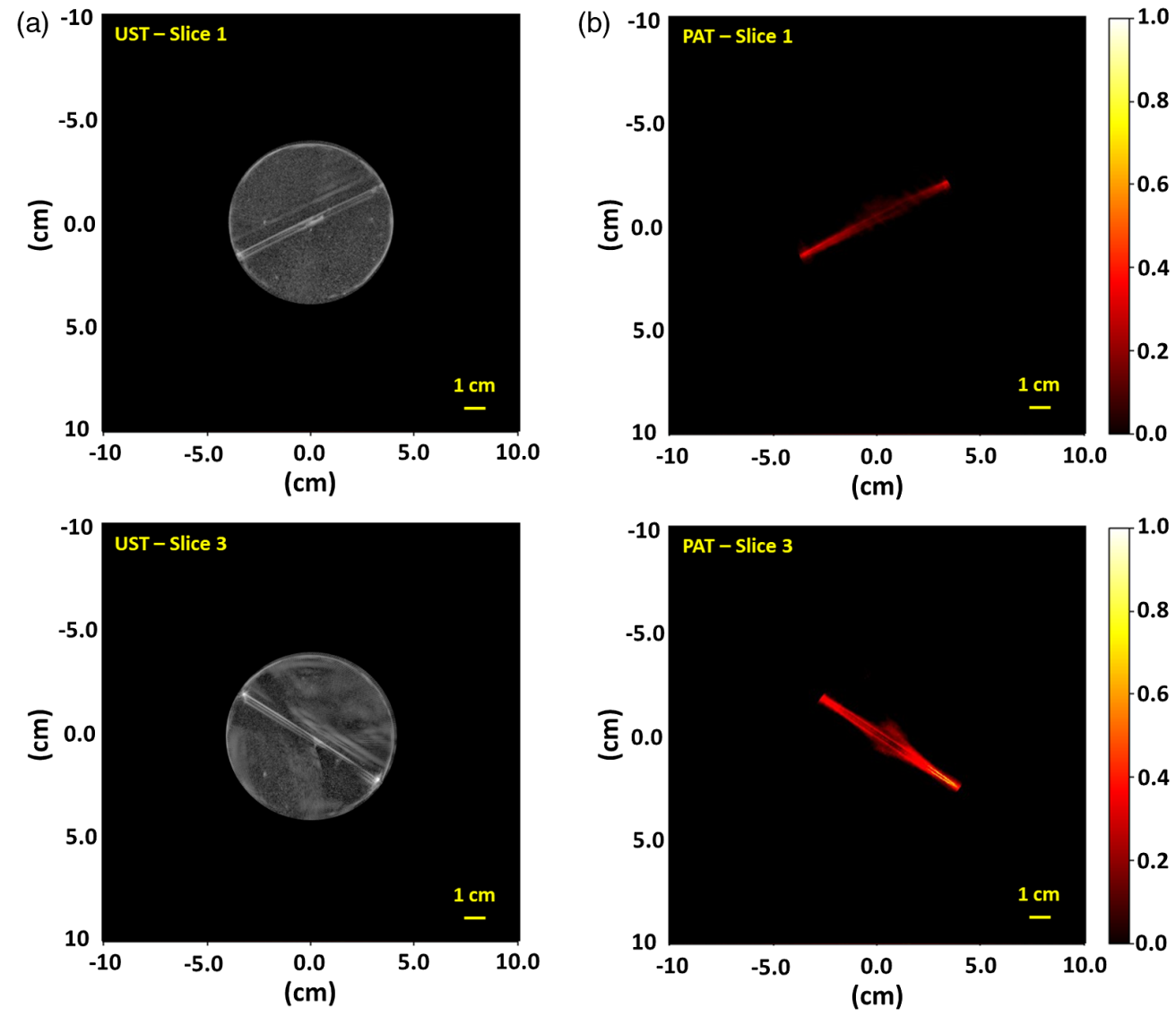

Fig. 5 (a) UST and (b) PAT images of slices 1 and 3, showing the 8-cm graphite absorber in the tissuemimicking phantom. The full-ring illumination is able to visualize the whole objects in all slices.

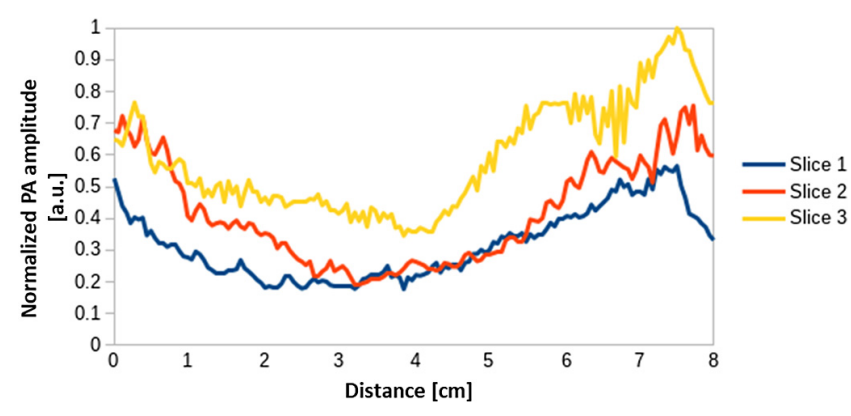

Fig. 6 Normalized PA amplitude across the 8-cm graphite object for all three cross-sectional slices, demonstrating the uniformity of the PA signal across the targeted cross-sectional slice.

to improve the PAT results. This system consists of a coneshaped reflector and two conical reflectors which can be adjusted independently for scanning the length of a cylindrical object. As shown in Fig. 7, the collimated beam from the laser source is directed normally to the cone-shaped reflector, and upon reflection, a circular beam is directed toward the stationary conical ring reflector. The stationary conical ring reflector then transmits the cylindrical-shaped beam to the mobile conical mirror which focuses the beam onto the targeted slice of the object. The cone-shaped reflector and the first stationary mirror are external to the water tank that houses the second mobile mirror and the ring US transducer.
During the scanning, the mobile conical reflector moves synchronously with the ring US transducer. Since the mobile reflector is placed below the transducer level, the breast tissue coverage will be identical to the UST and imaging areas close to the chest wall is feasible. The size and profile of the breast are defined by the UST images, which will be used to find the optimal position of the mobile reflector and adjust it to match the illumination and the acoustic acquisition planes. The design is verified by using ray tracing, which showed that it could illuminate breast tissue of $140 \mathrm{~mm}$ in diameter.

\section{Conclusions}

Multiple, proof-of-concept experiments using mirror-based, full-ring illumination systems were presented in this work for PAT imaging. A diagonal graphite absorber in gelatin and planar graphite absorbers were used to demonstrate uniformity of the illumination system, irrespective of vertical imaging depth. These experiments were followed by a full-ring illumination and full-ring US transducer system, which again demonstrated the energy uniformity within the phantom even with a non-optimal 39-deg illumination angle. Last, a proposed three-reflector, ring illumination US/PAT system was presented with a 66-deg illumination angle, which promises exciting future results for medical diagnosis due to its practicality of design and ease of scalability. All of the presented setups achieve a low fluence due to their inherent ring design and will no doubt benefit patients and clinicians in future imaging and diagnostic needs. 
(a)

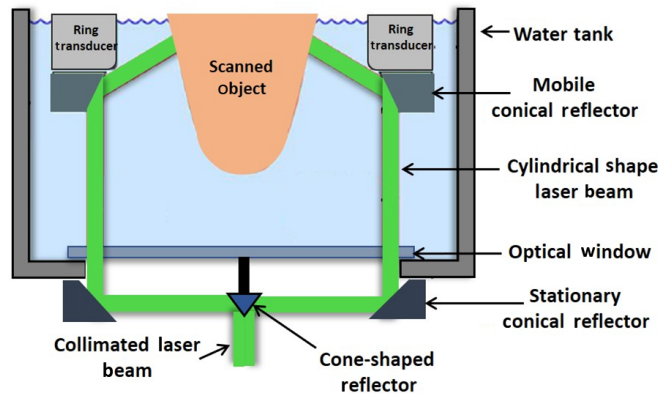

(b)

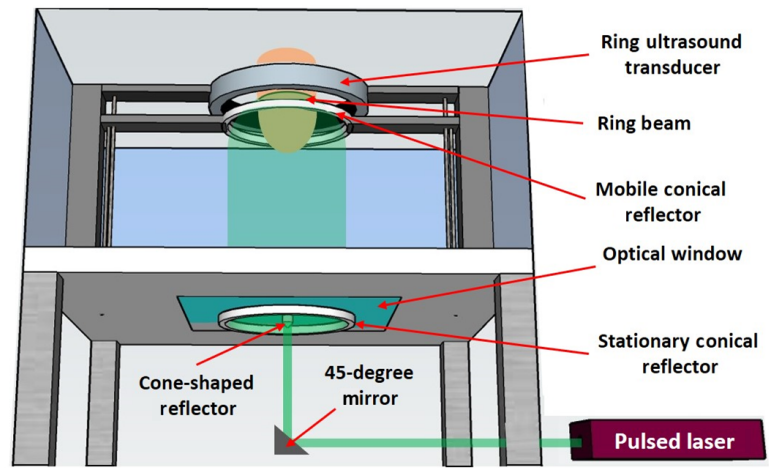

Fig. 7 (a) Cross-sectional view of the all-reflective, full-ring illumination and full-ring US transducer system, with three reflective elements in the imaging tank. (b) Three-dimensional view of the system showing a ring US-transducer, a pulsed laser, a cone-shaped reflector, and two conical reflectors.

\section{Disclosures}

The authors have no relevant financial interests in the manuscript and no other potential conflicts of interest to disclose.

\section{Acknowledgments}

The authors acknowledge Barbara Ann Karmanos Cancer Institute and Wayne State University for their financial support. Also, they would like to acknowledge Dr. Neb Duric from Karmanos Cancer Institute for his guidance and assistance in using the UST system. In addition, they would like to thank Ms. Sirisha Kondle, Mr. Barrington O'Brian, and Mr. Majeed Maghdeed from the Department of Electrical and Computer Engineering at Wayne State University for their assistance in conducting the study. MM is supported by Department of Defense (Breast Cancer Research program, Award No. (W81XWH-18-1-0039), University Research Grant from Wayne State University Provost office, Technology Development Incubator Award through Wayne State University, and pilot research funding from Barbara Ann Karmanos Cancer Institute. MAA is supported in part by Grant No. NSF DMS 1614305.

\section{References}

1. F. Bray et al., "Global cancer statistics 2018: GLOBOCAN estimates of incidence and mortality worldwide for 36 cancers in 185 countries," CA Cancer J. Clin. 68(6), 394-424 (2018).

2. J. G. Elmore et al., "Screening for breast cancer," JAMA 293(10), 12451256 (2005).

3. C. K. Kuhl et al., "Mammography, breast ultrasound, and magnetic resonance imaging for surveillance of women at high familial risk for breast cancer," J. Clin. Oncol. 23(33), 8469-8476 (2005).

4. R. M. Tamimi et al., "Endogenous hormone levels, mammographic density, and subsequent risk of breast cancer in postmenopausal women," J. Natl. Cancer Inst. 99(15), 1178-1187 (2007).

5. D. Saslow et al., "American cancer society guidelines for breast screening with MRI as an adjunct to mammography," CA Cancer J. Clin. 57(2), 75-89 (2007).

6. C. H. Lee et al., "Breast cancer screening with imaging: recommendations from the Society of Breast Imaging and the ACR on the use of mammography, breast MRI, breast ultrasound, and other technologies for the detection of clinically occult breast cancer," J. Am. Coll. Radiol. 7(1), 18-27 (2010).

7. J. A. Jensen, "Medical ultrasound imaging," Prog. Biophys. Mol. Biol. 93(1), 153-165 (2007).
8. P. B. Gordon and S. L. Goldenberg, "Malignant breast masses detected only by ultrasound. A retrospective review," Cancer 76(4), 626-630 (1995).

9. H. Madjar, "Role of breast ultrasound for the detection and differentiation of breast lesions," Breast Care 5(2), 109-114 (2010).

10. V. Corsetti et al., "Breast screening with ultrasound in women with mammography-negative dense breasts: evidence on incremental cancer detection and false positives, and associated cost," Eur. J. Cancer 44(4), 539-544 (2008).

11. N. Duric et al., "Breast imaging with the SoftVue imaging system: First results," Proc. SPIE 86750K (2013).

12. N. Duric et al., "Detection of breast cancer with ultrasound tomography: first results with the computed ultrasound risk evaluation (CURE) prototype," Med. Phys. 34(2), 773-785 (2007).

13. A. B. Karpiouk et al., "Combined ultrasound and photoacoustic imaging to detect and stage deep vein thrombosis: phantom and ex vivo studies," J. Biomed. Opt. 13(5), 054061 (2008).

14. B. Ranger et al., "Breast ultrasound tomography versus MRI for clinical display of anatomy and tumor rendering: preliminary results," Am. $J$. Roentgenol. 198(1), 233-239 (2012).

15. N. Duric et al., "Development of ultrasound tomography for breast imaging: technical assessment," Med. Phys. 32(5), 1375-1386 (2005).

16. S. Mallidi, G. P. Luke, and S. Emelianov, "Photoacoustic imaging in cancer detection, diagnosis, and treatment guidance," Trends Biotechnol. 29(5), 213-221 (2011).

17. M. Mehrmohammadi et al., "Photoacoustic imaging for cancer detection and staging," Curr. Mol. Imaging 2(1), 89-105 (2013).

18. R. A. Kruger et al., "Photoacoustic angiography of the breast," Med. Phys. 37(11), 6096-6100 (2010).

19. J. Menke, "Photoacoustic breast tomography prototypes with reported human applications," Eur. Radiol. 25(8), 2205-2213 (2015).

20. S. Manohar et al., "The Twente photoacoustic mammoscope: system overview and performance," Phys. Med. Biol. 50(11), 2543-2557 (2005).

21. R. A. Kruger et al., "Dedicated 3D photoacoustic breast imaging," Med. Phys. 40(11), 113301 (2013).

22. M. Klosner et al., "Advanced laser system for 3D optoacoustic tomography of the breast," Proc. SPIE 9708, 97085B (2016).

23. A. A. Oraevsky et al., "Laser optoacoustic imaging of breast cancer in vivo," Proc. SPIE 4256, 6-15 (2001).

24. S. A. Ermilov et al., "Laser optoacoustic imaging system for detection of breast cancer," J. Biomed. Opt. 14(2), 024007 (2009).

25. American Standard, Z136. 1. American National Standard for the Safe Use of Lasers, American National Standards Institute, Inc., New York (1993).

26. Z. Deng et al., "Acoustically penetrable optical reflector for photoacoustic tomography," J. Biomed. Opt. 18(7), 070503 (2013).

27. Z. Deng and C. Li, "Noninvasively measuring oxygen saturation of human finger-joint vessels by multi-transducer functional photoacoustic tomography," J. Biomed. Opt. 21(6), 061009 (2016).

28. Z. Deng, W. Li, and C. Li, "Slip-ring-based multi-transducer photoacoustic tomography system," Opt. Lett. 41(12), 2859-2862 (2016). 
29. J. Xia et al., "Whole-body ring-shaped confocal photoacoustic computed tomography of small animals in vivo," J. Biomed. Opt. 17(5), 050506 (2012).

30. L. Li et al., "Single-impulse panoramic photoacoustic computed tomography of small-animal whole-body dynamics at high spatiotemporal resolution," Nat. Biomed. Eng. 1(5), 0071 (2017).

31. S. Alshahrani et al., "An advanced photoacoustic tomography system based on a ring geometry design," Proc. SPIE 10580, 1058005 (2018).

32. S. Alshahrani et al., "Design and development of a full-ring ultrasound and photoacoustic tomography system for breast cancer imaging," in IEEE Int. Ultrasonics Symp. (IUS), pp. 1-1 (2017).

33. S. Alshahrani et al., "The Effectiveness of the omnidirectional illumination in full-ring photoacoustic tomography," in IEEE Int. Ultrasonics Symp. (IUS), pp. 1-4 (2018).

34. C. Li et al., "Breast imaging using waveform attenuation tomography," Proc. SPIE 10139, 101390A (2017).

35. M. Xu and L. V. Wang, "Universal back-projection algorithm for photoacoustic computed tomography," Phys. Rev. E 71(1), 016706 (2005).

Suhail Salem Alshahrani is a PhD student at Wayne State University (WSU), Department of Biomedical Engineering. He received his BSc degree in biomedical technology from King Saud University, College of Applied Medical Sciences, in 2008. Also, he received his master's degree in biomedical engineering from WSU, in 2014. His current research interests include photoacoustic and ultrasound tomography.

Yan Yan is a PhD candidate in the Biomedical Engineering Department at WSU, where he works on photoacoustic imaging device and reconstruction algorithm development. He obtained his BSc degree from Chong Qing University in posts and telecommunications and his MSc degree from WSU in computer science. The focus of his research is on the implementation of an integrated photoacoustic and ultrasound imaging device to improve fetal and maternal health care during pregnancy.

Naser Alijabbari received his BS degree in electrical engineering from the University of Maine, Orono, in 2005, and his PhD in electrical engineering from the University of Virginia, Charlottesville, in 2014. His research interests include the development of innovative point of care technology for improving the quality of healthcare and frugal system design.

Alexander Pattyn is a PhD student at WSU, Department of Biomedical Engineering. He received his BS degree in biomedical physics from WSU, College of Liberal Arts and Sciences, in 2017.
His current research interests include imaging physics, computational physics, and photoacoustic and ultrasound tomography.

Ivan Avrutsky is an associate professor of electrical/computer engineering, with adjunct appointment in physics/astronomy, WSU. Before joining WSU, he was postdoctoral fellow at Nortel Institute for Emerging Technologies at University of Toronto, and senior research fellow at the General Physics Institute of USSR Academy of Sciences. He holds his MS degree in laser physics from Moscow Institute of Physics and Technology, and his PhD in physics and mathematics from the General Physics Institute.

Eugene Malyarenko is a senior research scientist at Tessonics Corp, Birmingham, Michigan. He received his BS and MS degrees in physical acoustics from Kiev Taras Shehvchenko University, Ukraine, in 1994, and his PhD in ultrasonic nondestructive evaluation from the College of William and Mary, Virginia, in 2000. His research interests are in the areas of industrial and biomedical ultrasound, including pulmonary and dental diagnostics, skin and transcranial ultrasound imaging, small animal tomography, radiation dosimetry, and photoacoustics.

Joemini Poudel is a PhD candidate at Washington University in St. Louis. His research focus includes developing image reconstruction algorithms for medical imaging applications.

Mark Anastasio is the Donald Biggar Willett Professor in engineering and head of the Department of Bioengineering at the University of Illinois at Urbana-Champaign. He is an internationally recognized expert on computational imaging science, tomographic image reconstruction, and the development of novel computed biomedical imaging systems. His current research projects include the development of machine learning methods for optimizing imaging system performance and the development of image reconstruction methods for photoacoustic and ultrasound tomography.

Mohammad Mehrmohammadi is an assistant professor of biomedical/electrical and computer engineering at Wayne State University. $\mathrm{He}$ received his $\mathrm{BSc}$ degree in electrical engineering from Sharif University of Technology and his $\mathrm{PhD}$ in biomedical engineering from the University of Texas at Austin. Prior to joining Wayne State University, he worked at Mayo Clinic College of Medicine as a senior research fellow. His current research includes development of novel ultrasound, elasticity and photoacoustic imaging devices and techniques for diagnostic and monitoring of therapy procedures in various applications such as early stage cancer detection, fetal and maternal care, and guidance of vascular surgery procedures. 